\title{
Design of a Fast Handoff Scheme for Real-Time Media Application on the IEEE 802.11 Wireless LAN*
}

\author{
Mikyung Kang ${ }^{1}$, Junghoon Lee ${ }^{1, \star \star}$, Jiman Hong ${ }^{2}$, and Jinhwan Kim \\ ${ }^{1}$ Dept. of Computer Science and Statistics, Cheju National University, \\ ${ }^{2}$ School of Computer Science and Engineering, \\ Kwangwoon University \\ ${ }^{3}$ Dept. of Multimedia Engineering, Hansung University, \\ 690-756, Jeju Do, Republic of Korea \\ \{mkkang, jhlee\}@cheju.ac.kr, gman@daisy.kw.ac.kr, kimjh@hansung.ac.kr
}

\begin{abstract}
This paper proposes and analyzes a fast handoff scheme that exploits the overallocated bandwidth inevitably generated to guarantee the QoS requirement of real-time multimedia stream on the IEEE 802.11 wireless LAN. By using the reserved but not used network time and making the priority of the probe frame higher than any other data frames, $\mathrm{AP}$ and station can exchange RTS/CTS to negotiate when to send probe message, making AP immediately respond to the probe request with probe response message in the CFP. The result of simulation that focuses on the effect of the amount of overalloation and average number of simultaneous requests, shows that the proposed scheme reduces the AP scan time maximally by $16 \%$ for the given experiment parameters.
\end{abstract}

\section{Introduction}

IEEE 802.11 based WLANs(Wireless Local Area Networks) have seen immense growth in the last few years. Because of the mobility-enabling nature of wireless networks, there is an opportunity for many promising multimedia and peer-topeer applications such as VoIP, 802.11 phones and mobile video conferencing [1]. However, mobile clients suffer from quality degradation resulted from frequent handoff since each cell may cover just a small area, i.e., rooms or sections of a highway. Frequent handoffs and disconnections incur disruptions and instability of the connection between mobile host and server, even in the middle of an application session. To the worse, according to the handoff procedure defined in WLAN standard, the network connection as perceived by the application may be affected by the jittery and unpredictable handoff latencies. Such problem is particularly serious for the fast moving device such as telematics.

\footnotetext{
* This research was supported by the MIC, Korea, under the ITRC support program supervised by the IITA.

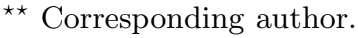


Wireless LAN STA (Station) is the most basic component of the wireless network 2] and it means any device that contains the functionality of the 802.11 protocol. BSS (Basic Service Set) is the basic building block of an 802.11 WLAN, and each BSS consists of any number of stations. As a SSID (Service Set IDentifier) is a unique identifier that distinguishes one WLAN from the others, all APs (Access Points) and STAs attempting to join a specific WLAN must have the same SSID. A WLAN handoff is performed at the MAC layer when a mobile STA moves beyond the radio range of the current AP and enters another BSS 3 . During the handoff, management frames are exchanged between the STA and the AP. The handoff procedure essentially requires the transfer of STA information such as authentication, authorization, and accounting information, from the old AP to the new AP.

Previous research results show that the probe phase overwhelms the total handoff latency while the variation in the probe-wait time also causes the large variations in the overall handoff latency. For the client, the service is ceased during the handoff. Because the STA must scan the channel to which an AP may belong for the maximum scanning period, and it must repeat iteratively for all channels, the probe time occupies the biggest part of the handoff latency. Thus any handoff scheme built upon the techniques/heuristics that either cache or deduce AP information without having to actually perform a complete active scan definitely should cope with the dominating cost of the scan process.

To solve such a problem by reducing AP scanning delay of handoff latency at MAC layer, this paper proposes and analyzes a fast handoff scheme that exploits the overallocated bandwidth necessarily accompanied in providing QoS guarantee to real-time multimedia stream. Using the reserved but not used network time, AP and STA can exchange RTS (Request To Send)/CTS (Clear To Send) to negotiate when to send a probe message, making AP immediately respond to the probe request with matching probe response message in the next CFP. In addition, by making the priority of the probe frame higher than any other data frames, collision between probe response messages and ordinary data frames can be eliminated, and dynamic adjustment of the channel scan time further improves AP scanning time. With the reduced AP scan time, a seamless handoff process is performed, minimizing the deadline miss ratio.

This paper is organized as follows: After issuing the problem in Section 1, Section 2 introduces the related works. With the description on the handoff procedure in IEEE 802.11 WLANs in Section 3, Section 4 proposes the fast handoff scheme. Section 5 shows and discusses the performance measurement results and then Section 6 finally concludes this paper with a brief summarization and the description of future works.

\section{Related Works}

A lot of works have been already carried out to reduce the handoff latency for the roaming client station. However, existing handoff schemes are not suitable for meeting requirements of real-time multimedia application due to its long 
and occasionally unbounded delay. The sequence of messages being exchanged during the handoff process can be categorized into three groups, namely, probe, authentication, and association. Accordingly, existing works are also classified by the delay element to reduce.

First, the researches to improve the probe delay are as follows: Kim et al. proposed a selective scanning algorithm using the neighbor graphs [4]. This approach forces changes in the network infrastructure and use of IAPP (Inter Access Point Protocol) though it narrows the search space with neighbor graphs. Moreover, this scheme does not consider the time amount required by the client to process the received probe responses. Shin et al. proposed a new handoff procedure which reduces the MAC layer handoff latency, in most cases, to a level where VoIP communication becomes seamless using both selective scanning algorithm and caching mechanism [2. It needs just an insignificant modification in the clientside wireless card driver such as channel mask and improved cache dimensioning. According to the analysis result by Jain [5] and Mishra 3], there are remarkable variations in handoff latencies with change in SSID and channel of APs, and probe delay is the major malicious factor to the total handoff performance.

Second, to improve the authentication delay, Pack et al. proposed a fast InterAP handoff scheme using the predictive authentication method based on IEEE 802.1x model 6] 7]. The IEEE 802.1x authentication delay is reduced by using the FHR (Frequent Handoff Region) selection algorithm that makes the candidate APs selected by the predictive algorithm, perform the pre-authentication, directly taking into account traffic patterns and user characteristics, which are collected and managed in the centralized system.

Third, to improve the association delay, Mishra et al. focused on reducing the reassociation delay [8]. The reassociation delay is reduced by using a caching mechanism on the AP side. This caching mechanism is based on the IAPP protocol in order to exchange the client context information between neighboring APs. The cache in the AP is built by observing the information contained in an IAPP Move-Notify message or in the reassociation request sent to the AP by the client. By exchanging the client information with the old AP, the new AP prevents the client from sending its context information, resulting in the reduction of the reassociation delay.

\section{Backgrounds}

\subsection{IEEE 802.11 WLAN}

The wireless LAN operates on both CP (Contention Period) and CFP (Contention Free Period) phases alternatively in BSS as shown in Fig. 1(a). Each superframe consists of CFP and CP, which are mapped to PCF (Point Coordination Function) and DCF (Distributed Coordination Function), respectively [9]. Though PCF is optional, QoS guarantee cannot be provided without PCF. Recently, the PCF-enabled schemes are increasingly being applied to the WLAN showing a reasonable throughput. Moreover, previous researches based on the 
DCF can not avoid both the collision between the probe messages and normal data frames, and the probe delay according to the backoff time of DCF.

Fig. 1(a) shows the operation of polling procedure as well as the allocation of capacity vector. The DCF exploits collision-based CSMA/CA (Carrier Sense Multiple Access with Collision Avoidance) protocol for non-real-time messages, and RTS/CTS clearing technique to further reduce the possibility of collisions as shown in Fig. 1(b).

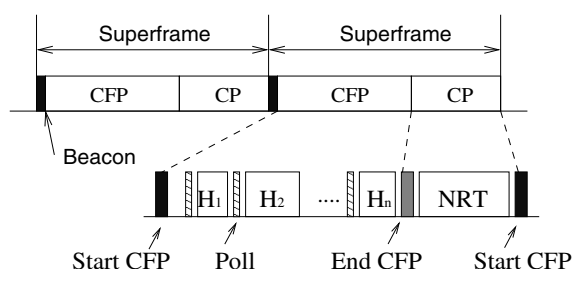

(a) Time axis of wireless LAN

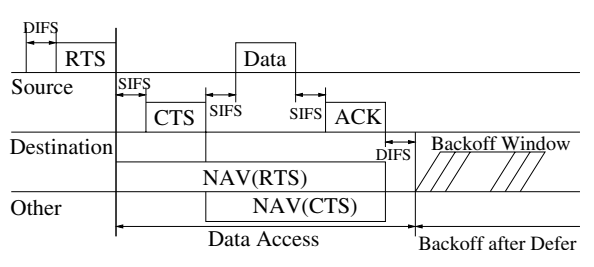

(b) RTS/CTS frame exchange

Fig. 1. IEEE 802.11 WLAN operations

PC (Point Coordinator) node, typically AP, sequentially polls each station during CFP. AP maintains the polling list ordered by a polling sequence. The PC attempts to initiate CFP by broadcasting a Beacon at regular intervals derived from a network parameter of CFPRate. The polled node transmits its message for a predefined time interval, and it always responds to a poll immediately whether it has a pending message or not. Only after the medium is idle the coordinator will get the priority due to the shorter IFS (InterFrameSpace).

\subsection{The Handoff Procedure in WLAN}

The handoff process can be divided into two logical steps of discovery and reassociation $[3$. The discovery process involves handoff initiation and scanning phases. As signal strength and signal-to-noise ratio from a station's current AP get weaker, STA loses connectivity and initiates a handoff. Then the client is not able to communicate with its current AP, so the client needs to find the other APs available. This scan function is performed at a MAC layer, and the station can create the available AP list ordered by the received signal strength.

For the scan phase, STA can perform scan operation either in passive or active mode. In passive scan mode, using the information obtained from beacon frames, STA listens to each channel of the physical medium to try and to locate an AP. In the active mode (the wireless NICs do by default), as shown in Fig. 2(a), STA broadcasts additional probe packets on each channel and receives responses from APs. Thus the STA actively probes for the APs, and the actual number of messages varies from 3 to 11 . Fig. 2(b) shows the sequence of messages typically observed during a handoff process. The handoff process starts with the first probe request and ends with a reassociation response from the new $\mathrm{AP}$. 


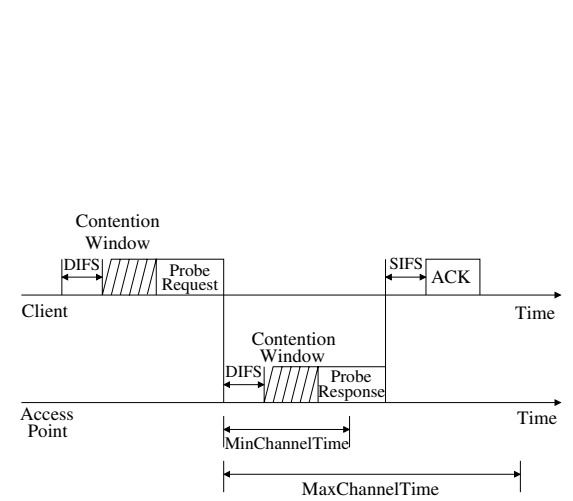

(a) Active Scan

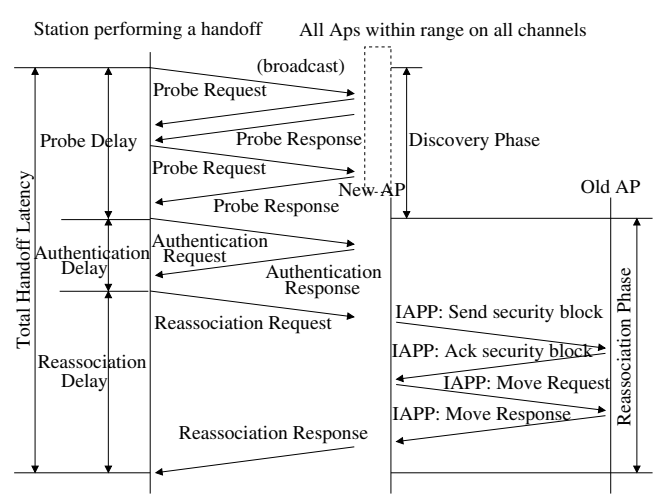

(b) WLAN Handoff Procedure

Fig. 2. Handoff procedures

The probe function follows the IEEE 802.11 MAC active scan function and the standard specifies a scanning procedure as follows [2] [3]:

1. Using CSMA/CA, acquire the access right to the medium.

2. Transmit a probe request containing the broadcast address as destination, SSID, and broadcast BSSID (Basic SSID).

3. Start a ProbeTimer.

4. If medium is not busy before the ProbeTimer reaches MinChannelTime, scan the next channel. Otherwise, process all received probe responses.

5. Move to next channel and repeat the above steps.

After all channels have been scanned, informations received from probe response are scrutinized by STA to select a new AP. Once the STA decides to join a specific AP, authentication messages are exchanged between the STA and the selected AP, and after a successful authentication, the STA sends a reassociation request and expects a reassociation response back from the AP.

\section{Proposed Scheme}

\subsection{AP Channel Management}

Real-time guarantee is provided based on the worst case available transmission time, so a stream can meet extra slots in some periods. Moreover, as $C_{i}$ is usually just the upper bound of message size in multimedia applications, some period, $P_{i}$, has message to send less than $C_{i}$. As a result, a node possibly has no pending message when it receives a poll. Though there have been plenty of bandwidth allocation schemes for the real-time message stream or sensor data stream, we exploit Lee's scheme, as it completely conforms to WLAN standard [10].

To begin with, let $\delta$ denote the total overhead of a superframe including polling latency, IFS and the like, while $D_{\max }$ the maximum length of a data 


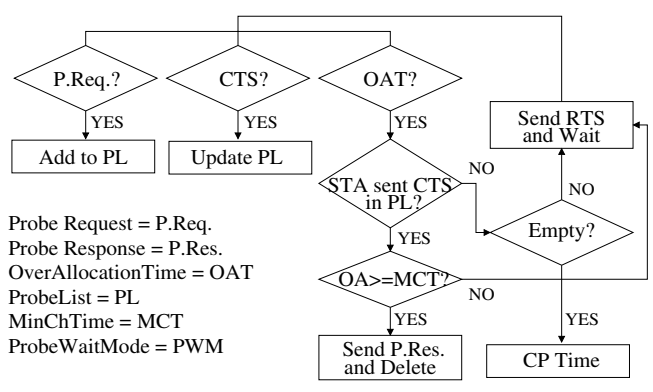

(a) AP Process

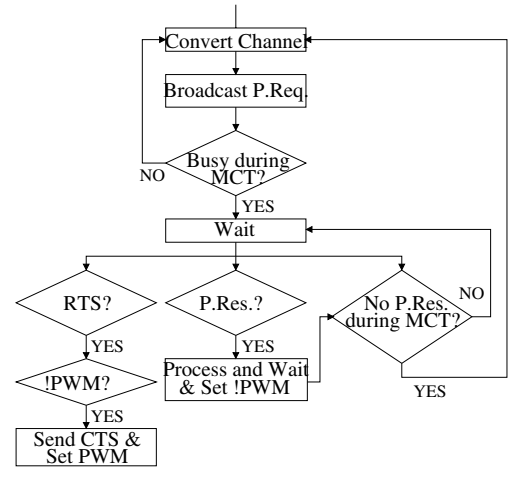

(b) STA Process

Fig. 3. AP and STA processes

packet. For each superframe, at least a time amount as large as $D_{\max }$, should be reserved for a data packet so as to keep compatibility with WLAN standard. The capacity vector, $H_{i}$, is large enough to exchange RTS/CTS. If $P_{\min }$ is the smallest element of set $\left\{P_{i}\right\}$, the requirement for the superframe time, $F$, can be summarized as follows:

$$
\begin{array}{ll}
H_{i}=\frac{C_{i}}{\left(\left\lfloor\frac{P_{i}}{F}\right\rfloor-1\right)} & \text { if }\left(P_{i}-\left\lfloor\frac{P_{i}}{F}\right\rfloor \cdot F\right) \leq D_{\max } \\
H_{i}=\frac{C_{i}}{\left\lfloor\frac{P_{i}}{F}\right\rfloor} & \text { Otherwise }
\end{array}
$$

By this, we can determine the length $\mathrm{CFP}$ period $\left(T_{C F P}\right)$ and that of $\mathrm{CP}\left(T_{C P}\right)$ as follows:

$$
T_{C F P}=\sum H_{i}+\delta, \quad T_{C P}=F-T_{C F P} \geq D_{\max }
$$

For detailed description, refer to [10].

\subsection{AP Scanning Procedure}

In general, it takes more time to perform a passive scan than active scan in collecting the necessary information. Thus current WLAN equipments use active scan mode in order to reduce handoff delay. In the standard active scanning procedure, though the probe phase of one channel can be terminated before the ProbeTimer reaches MaxChannelTime, the STA has to wait during the MaxChannelTime. In addition, the probe response messages and other ordinary data frames contend for the shared channel, so they can collide with one another. To minimize the collision using active scan mode, we will assign the higher priority to the probe response message, and also provide variable scan time.

As shown in Fig. 3, the proposed AP scanning scheme is performed through the unused slots. AP can not only send RTS message to the STA which waits for probe response message, but also STA can respond with CTS message to the appropriate AP which will transmit the probe response message. Once the RTS/CTS messages are exchanged, the priority to send probe response message 
is assigned to the AP, and other APs can not receive the CTS message until the selected AP sends probe message to the STA.

The procedure of the proposed scheme using adaptive scan time is as follows:

1. Using CSMA/CA, STA acquires the access right to the medium.

2. STA transmits a probe request frame containing the broadcast address as destination, SSID, and broadcast BSSID, to all APs in the reachable channels. And AP is informed the existence of a STA that waits to join.

3. Start a ProbeTimer.

4. If medium is not busy before the Probe Timer reaches MinChannelTime, STA scans the next channel. Otherwise, following steps are applied.

5. Using the unused slot, AP sends RTS to STA specifying the ID submitted in step 2.

6. STA responses with CTS if it still wants to receive the probe response.

7. AP sends probe response to STA if overallocated slot time amount is large enough to send the probe message. Otherwise, probe response is postponed until AP meets such a slot.

8. If the number of RTS messages is equal to the number of probe responses, and if SSID of being transmitted packet is equal to that of already received probe response during the MinChannelTime, that is, after sensing that the probe response doesn't arrive at STA any more, STA scans the next channel.

\section{Performance Measurements}

This section will show performance measurement results performed via simulation. The experiments are based on some assumptions to concentrate on the major performance parameters, namely, the amount of overallocation and the number of pending handoff request. Every stream has equal period and communication time, while each time variable is aligned to $F$ and total number of streams is set to 5 for simplicity. However, these assumption can be easily generalized into the more realistic circumstances. Finally, we compared the handoff time of

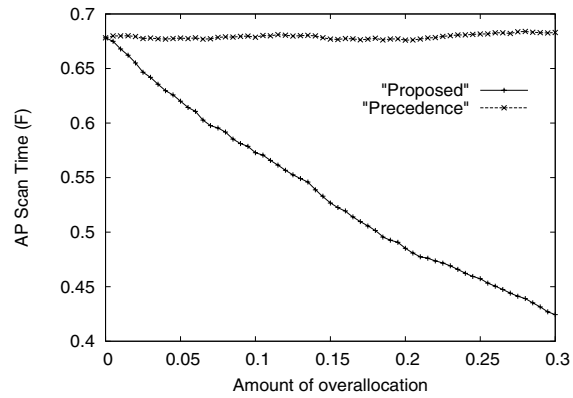

Fig. 4. Scan time vs. overallocation

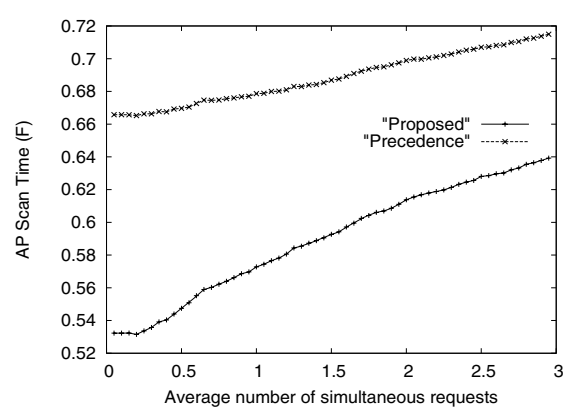

Fig. 5. Scan time vs. \# of requests 
the proposed scheme with that of a scheme which just gives the precedence to the packets relevant to handoff procedure.

Fig. 4 shows the effect of overallocated bandwidth to the AP scan time. The probability of unused slot due to overallocation can be estimated as $\left(\frac{H_{i}}{F}-\frac{C_{i}}{P_{i}}\right)$. The efficient usage of overallocated bandwidth can speed up the handoff time by $16 \%$ when the overallocation value is 0.1 . Fig. 5 also plots the AP scan time according to the number of simultaneous requests. The performance gap gets narrow when more handoff requests are submitted to the network as the proposed scheme can expect the improvement only if CFP has an overallocation larger than the handoff procedure. The total AP scan time can be calculated by the sum of time needed to scan a used channel and time to scan on empty channel.

\section{Conclusion and Future Work}

This paper proposes and analyzes a fast handoff scheme that exploits the overallocated bandwidth inevitably generated to guarantee the QoS requirement of real-time multimedia stream on the IEEE 802.11 Wireless LANs. Using the reserved but not used network time, AP and STA can exchange RTS/CTS to negotiate when to send probe message, making AP immediately respond to the probe request with probe response message in the next CFP. In addition, by making the priority of the probe frame higher than any other data frames, collision of probe response messages and ordinary data frames can be minimized. Simulation results show that the proposed scheme improves the AP scan time according to the amount of overalloation and average number of simultaneous requests.

The channel with the best signal is not necessarily the best channel to connect to because it could be much more congested than a channel with a lower signal strength. Because of this, a heuristic which considers bit rate information together with signal strength can achieve optimal performance. As a future work, we consider an error control mechanism as well as a heuristic algorithm that signal strength can achieve optimal performance.

\section{References}

1. Mao, S., Lin, S., Wang, Y., Panwar, S. S., Li, Y.: Multipath video transport over wireless ad hoc networks. IEEE Wireless Communications (2005)

2. Shin, S., Rawat, A., Schulzrinne, H.: Reducing MAC Layer Handoff Latency in IEEE 802.11 Wireless LANs. ACM MobiWac'04 (2004)

3. Mishra, A., Shin, M., Arbaugh, W.: An Empirical Analysis of the IEEE 802.11 MAC Layer Handoff Process. ACM Computer Communications Review, Vol. 33, No.2 (2003) 93-102

4. Kim, H. Park, S. Park, C, Kim, J., Ko, S.: Selective channel scanning for fast handoff in wireless lan using neighbor graph. International Technical Conference of Circuits/Systems, Computer and Communications (2004)

5. Jain, A.: Handoff Delay for 802.11b Wireless LANs, Project Report (2003) 
6. Pack, S., Choi, Y.: Fast Inter-AP Handoff using Predictive-Authentication Scheme in a Public Wireless LAN. IEEE Networks (2002)

7. Pack, S., Choi, Y.: Pre-Authenticated Fast Handoff in a Public Wireless LAN based on IEEE 802.1x Model. IFIP TC6 Personal Wireless Communications (2002)

8. Mishra, A., Shin, M., Arbaugh, W.: Context Caching using Neighbor Graphs for Fast Handoffs in a Wireless Network. IEEE INFOCOM (2004)

9. IEEE Part 11: Wireless LAN Medium Access Control (MAC) and Physical Layer (PHY) Specifications. IEEE Standard 802.11 (1999)

10. Lee, J., Kang, M., Jin, Y., Kim, H., Kim, J.: An efficient bandwidth management scheme for a hard real-time fuzzy control system based on the wireless LAN. Lecture Notes in Artificial Intelligence, Vol. 3642. Springer-Verlag, Berlin Heidelberg New York (2005) 644-659 